\section{Neumonía nosocomial en el postoperatorio de la cirugía cardiaca: ¿puede realmente estar bajo nuestro control?}

\section{Postoperative pneumonia after cardiac surgery: Really, can it be under our control?}

Los pacientes sometidos a cirugía cardiaca, representan una población de alto riesgo para el desarrollo de infecciones nosocomiales en el período postoperatorio ${ }^{1}$. Algunas series incluso reportan hasta más de un $20 \%$ de incidencia ${ }^{2}$, y son causa de incremento en la mortalidad, la estancia hospitalaria, el uso de antibióticos y los costos de atención. La mortalidad en la $\mathrm{UCl}$ atribuible a neumonía asociada a la ventilación (NAV) puede llegar a ser hasta del 45\% Dada la importancia del tema y el amplio trabajo por hacer en este campo hemos leído con gran interés el trabajo llevado a cabo por Vera Urquiza et al. ${ }^{5}$ y consideramos que para una adecuada extrapolación clínica de estos resultados, sería recomendable puntualizar algunos aspectos que detallamos.

Desde el punto de vista metodológico, en los criterios de inclusión no se definen claramente los criterios diagnósticos de neumonía que se utilizaron, para poder determinar cuáles de estos corresponderían a NAV y cuáles a neumonía nosocomial, esto es fundamental para estandarizar, documentar qué episodios se presentaron realmente, y así poder identificar medidas correctivas y preventivas. Por otro lado en el diagnóstico se utiliza como punto de corte para un conteo bacteriano significativo $1 \times 10^{3} \mathrm{UFC} / \mathrm{ml}$, pero no se explica el tipo de muestra respiratoria utilizada (aspirado bronquial, lavado bronquioalveolar, lavado con cepillo protegido).

Una adecuada clasificación de la gravedad es importante, y por este motivo consideramos relevante el que no aparezca en el texto el EUROSCORE de los pacientes, fundamental para evaluar de forma estándar el riesgo quirúrgico y la severidad de los pacientes que se están sometiendo a cirugía cardiaca.

En la fase de extubación, en el texto se menciona que el tiempo promedio de extubación fue de $58 \mathrm{~h}$, pero en las características postoperatorias de la tabla 1 se indica que el tiempo de extubación en horas fue en promedio de $0.77 \mathrm{~h}$. El tiempo de extubación constituye un factor de riesgo importante para neumonía en el postoperatorio de cirugía cardiaca ya reportado en otras series y revisiones ${ }^{6,7}$, por lo que es muy relevante en este trabajo. $\mathrm{Y}$ un tiempo promedio de $58 \mathrm{~h}$ pareciera importante en un contexto donde los pacientes tienen un promedio de la FE del $58 \%$ y un perfil general de los pacientes para un EUROSCORE estimado de 2 que constituye bajo riesgo.
Llama la atención la HTA como factor de riesgo en el análisis multivariado, podría quizás explicarse por un porcentaje importante de hipertensos en la población estudiada casi del 40\% (lo cuál es esperable), y el tamaño de la población estudiada (pequeña), podrían jugar un papel.

Consideramos que es necesario realizar un análisis más profundo de los aspectos que se relacionan con las complicaciones asociadas a la cirugía cardiaca, teniendo en cuenta estos comentarios.

\section{Bibliografía}

1. Hortal J, Maddalena G, Perez MJ, et al. Incidence and risk factors for ventilator associated pneumonia after major heart surgery. Intensive Care Med. 2009;35:1518-25.

2. Segers P, Speekenbrink R, Ubbink $D$, et al. Prevention of nosocomial infection in cardiac surgery by decontamination of the nasopharynx and oropharynx with chlorhexidine gluconate. JAMA. 2006;296:2460-6.

3. Fagon JY, Chastre J, Hance AJ, et al. Nosocomial pneumonia in ventilated patients: A cohort study evaluating attributable mortality and hospital stay. Am J Med. 1993;94:281-8.

4. Cunnion KM, Weber DJ, Broadhead WE, et al. Risk factors for nosocomial pneumonia: Comparing adult critical care populations. Am J Respir Crit Care Med. 1996;153:158-62.

5. Vera Urquiza R, Bucio Reta ER, Berríos Bárcenas EA, et al. Risk factors for the development of postoperative pneumonia after cardiac surgery. Arch Cardiol Mex. 2016;86:203-7.

6. He S, Chen B, Li W, et al. Ventilator-associated pneumonia after cardiac surgery: A meta-analysis and systematic review. J Thorac Cardiovasc Surg. 2014;148:3148-55.

7. García-Delgado M, Navarrete-Sánchez I, Colmenero M. Preventing and managing perioperative pulmonary complications following cardiac surgery. Curr Opin Anaesthesiol. 2014;27: 146-52.

Antonio M. Esquinas ${ }^{\mathrm{a}}$ y Julián Arias-Ortiz ${ }^{\mathrm{b}, *}$

a Unidad de Cuidados Intensivos, Hospital Morales

Meseguer, Murcia, España

b Unidad de Cuidados Intensivos Quirúrgicos, Hospital

Rafael Ángel Calderón Guardia, Universidad de Costa Rica, San José, Costa Rica

* Autor para correspondencia: Apartado postal 323-1007 San José, Costa Rica.

Correo electrónico: julian.ariasortiz@gmail.com

(J. Arias-Ortiz).

http://dx.doi.org/10.1016/j.acmx.2017.02.006

1405-9940/

(c) 2017 Instituto Nacional de Cardiología Ignacio Chávez. Publicado por Masson Doyma México S.A. Este es un artículo Open Access bajo la licencia CC BY-NC-ND (http: / / creativecommons.org/ licenses/by-nc-nd/4.0/). 